\title{
A Schinzel theorem on continued fractions in function fields
}

\author{
by \\ WEIQUN Hu (Nanjing)
}

1. Introduction and statement of result. It is well known that the expansion of the continued fraction of a positive quadratic irrational number $\alpha$ is periodical. The problem of evaluating and estimating the period $p(\alpha)$ of the continued fraction of $\alpha$ is closely related to many number theoretic problems such as Pell's equations and the fundamental unit of the real quadratic field $\mathbb{Q}(\alpha)$. In 1961, A. Schinzel [8] proved an interesting result. Let $a, b, c$ be integers with $a \geq 1$, and let $f(x)=a^{2} x^{2}+b x+c$ be a quadratic polynomial with discriminant $d=b^{2}-4 a^{2} c$. We define two sets

$$
\begin{gathered}
\widetilde{\mathbb{Z}}=\{n \in \mathbb{Z}: n \geq 1 \text { and } f(n) \text { is square-free }\}, \\
E=\left\{n \in \widetilde{\mathbb{Z}}: d \nmid\left(2 a^{2} n+b\right)^{2}\right\} .
\end{gathered}
$$

Schinzel's result says that

$$
\text { (I) } \varlimsup_{n \in \widetilde{\mathbb{Z}}-E} p(\sqrt{f(n)})<\infty, \quad \text { (II) } \varlimsup_{n \in E} p(\sqrt{f(n)})=\infty .
$$

Later, S. Louboutin [6] and A. Farhane [2] presented more exact and effective lower bounds of $p(\sqrt{f(n)})$ for $n \in E$. In this paper we show an analogue of Schinzel's result in the function field case.

Let $\mathbb{F}_{q}$ be the finite field with $q$ elements where $q$ is odd, $k=\mathbb{F}_{q}(t)$ be the rational function field over $\mathbb{F}_{q}, R=\mathbb{F}_{q}[t]$ be its polynomial ring, $k_{\infty}=\mathbb{F}_{q}((1 / t))$ be the completion of $k$ at the infinite place $\infty=(1 / t)$. Each element $\alpha \in k_{\infty}-k$ is a (formal) power series

$$
\alpha=\sum_{n \geq l} c_{n}\left(\frac{1}{t}\right)^{n}, \quad c_{n} \in \mathbb{F}_{q}(n \geq l), \quad \operatorname{sgn} \alpha=c_{l} \in \mathbb{F}_{q}^{*}
$$

Let $v_{\infty}$ be the normal exponential valuation in $k_{\infty}$ with $v_{\infty}(1 / t)=1$. In this paper we prefer to use the notation $\operatorname{deg} \alpha=-v_{\infty}(\alpha)=-l$ since it is

2000 Mathematics Subject Classification: 11D41, 11R27, 11R58.

Key words and phrases: continued fraction, function field. 
just the ordinary degree if $\alpha$ is a polynomial in $R$. We define

$$
[\alpha]=\sum_{n=l}^{0} c_{n}\left(\frac{1}{t}\right)^{n} \in R, \quad\{\alpha\}=\sum_{n \geq \sup \{1, l\}} c_{n}\left(\frac{1}{t}\right)^{n} .
$$

Then we have the continued fraction expansion of $\alpha$,

$$
\alpha=\left[a_{0}, a_{1}, a_{2}, \ldots\right]=a_{0}+\frac{1}{a_{1}+\frac{1}{a_{2}+\ddots} .}
$$

where $a_{0}=[\alpha]$ and $\alpha_{i}=1 /\left\{a_{i-1}\right\}, a_{i}=\left[\alpha_{i}\right]$ for $i \geq 1$ so that

$$
\alpha_{i}=\left[a_{i}, a_{i+1}, \ldots\right], \quad \operatorname{deg} \alpha_{i}=\operatorname{deg} a_{i} \geq 1 \quad \text { for } i \geq 1 .
$$

Suppose that $F(t)$ is a square-free polynomial in $R$ such that $\operatorname{deg} F(t) \geq 1$ and $\sqrt{F(t)} \in k_{\infty}$. (Note: $F(t)$ is called square-free if there is no $A \in R$ with $\operatorname{deg} A \geq 1$ such that $A^{2} \mid F(t)$. By Hensel's lemma, $\sqrt{F(t)} \in k_{\infty}$ if and only if $2 \mid \operatorname{deg} F(t)$ and $\operatorname{sgn} F(t) \in \mathbb{F}_{q}^{* 2}$.) The subfield $K=k(\sqrt{F(t)})$ of $k_{\infty}$ is called a "real" quadratic function field. It is well known that the continued fraction of $\sqrt{F(t)}$ is periodical. In fact, we need a generalized version of the period in the study of the algebraic structure of the real quadratic function field $K$. For each $\alpha \in k_{\infty}, \alpha$ is algebraic over $k$ with degree two if and only if the continued fraction of $\alpha=\left[a_{0}, a_{1}, \ldots, a_{n}, \ldots\right]$ is infinite and quasi-periodical, which means that there exist $c \in \mathbb{F}_{q}^{*}$ and integers $n_{0} \geq 0$ and $l \geq 1$ such that

$$
a_{n+l}=c^{(-1)^{n}} a_{n} \text { for all } n \geq n_{0} .
$$

The smallest positive integer $l$ satisfying this condition is called the quasiperiod of the continued fraction $\alpha=\left[a_{0}, a_{1}, \ldots\right]$ and denoted by $p(\alpha)$. If $\alpha=$ $\sqrt{F(t)}$ and $l=p(\sqrt{F(t)})$, then the fundamental unit of the real quadratic function field $K=k(\sqrt{F(t)})$ is $P_{l-1}+Q_{l-1} \sqrt{F(t)}$ where

$$
P_{l-1} / Q_{l-1}=\left[a_{0}, a_{1}, \ldots, a_{l-1}\right] .
$$

The continued fraction method of studying the ideal class number and units of real quadratic function fields was initiated by E. Artin [1]. For recent work we refer to D. Hayes [4] and C. D. González [3].

Now we state the result of this paper. Let $A, B, C$ be polynomials in $R=\mathbb{F}_{q}[t], \operatorname{deg} A \geq 0, f(x)=A^{2} x^{2}+B x+C, D=B^{2}-4 A^{2} C$, and

$$
\begin{aligned}
& \widetilde{R}=\left\{N \in R: f(N) \text { is square-free and } \sqrt{f(N)} \in k_{\infty}\right\}, \\
& E=\left\{N \in \widetilde{R}: D \nmid\left(2 A^{2} N+B\right)^{2}\right\} .
\end{aligned}
$$

Let $p(\sqrt{f(N)})$ be the quasi-period of the continued fraction of $\sqrt{f(N)}$. 


\section{THEOREM 1.1.}

$$
\varlimsup_{N \in \widetilde{R}-E} p(\sqrt{f(N)})<\infty
$$

THEOREM 1.2 .

$$
\lim _{N \in E} p(\sqrt{f(N)})=\infty .
$$

Before proving Theorems 1.1 and 1.2, in Section 2 we introduce several basic facts on continued fractions in function fields. Most results are just simple analogues of facts in the theory of ordinary continued fractions in the real number field case (see Hua's book [5], for instance), so we present the proof of some particular facts for the reader's convenience and omit the proof of others. In Sections 3 and 4 we prove Theorems 1.1 and 1.2.

2. Continued fractions in function fields. From now on we fix the following notations: $k=\mathbb{F}_{q}(t)(2 \nmid q), R=\mathbb{F}_{q}[t], k_{\infty}=\mathbb{F}((1 / t))$. For $\alpha \in$ $k_{\infty}-k$,

$$
\alpha=c_{m}\left(\frac{1}{t}\right)^{m}+c_{m+1}\left(\frac{1}{t}\right)^{m+1}+\ldots, \quad c_{i} \in \mathbb{F}_{q}(i \geq m), c_{m} \in \mathbb{F}_{q}^{*} ;
$$

we set $\operatorname{deg} \alpha=-m, \operatorname{sgn} \alpha=c_{n}$. We have the continued fraction

$$
\alpha=\left[a_{0}, a_{1}, \ldots, a_{n}, \ldots\right] .
$$

For $n \geq 0$, the $n$th convergent of the continued fraction (2.1) is

$$
P_{n} / Q_{n}=\left[a_{0}, a_{1}, \ldots, a_{n}\right]
$$

which can be calculated recursively by

$$
\begin{aligned}
& P_{0}=a_{0}, \quad P_{1}=a_{1} a_{0}+1, \quad P_{n}=a_{n} P_{n-1}+P_{n-2} \quad(n \geq 2), \\
& Q_{0}=1, \quad Q_{1}=a_{1}, \quad Q_{n}=a_{n} Q_{n-1}+Q_{n-2} \quad(n \geq 2) \text {. }
\end{aligned}
$$

We have the following basic facts.

FACT 1. For $n \geq 1$,

$$
\begin{gathered}
P_{n} Q_{n-1}-P_{n-1} Q_{n}=(-1)^{n+1}, \\
\alpha=\frac{\alpha_{n+1} P_{n}+P_{n-1}}{\alpha_{n+1} Q_{n}+Q_{n-1}}
\end{gathered}
$$

where $\alpha_{n}=\left[a_{n}, a_{n+1}, \ldots\right]$.

Let $A, B, C \in R, \operatorname{deg} A \geq 0, f(x)=A^{2} x+B x+C, D=B^{2}-4 A^{2} C$, and $\sqrt{f(N)} \in k_{\infty}(N \in R)$.

FACT 2. For $\alpha=\sqrt{f(N)}=\left[a_{0}, a_{1}, \ldots, a_{n}, \ldots\right]$, we have

$$
\alpha_{n}=\frac{U_{n}+\sqrt{f(N)}}{V_{n}} \quad(n \geq 0)
$$


where $U_{n}, V_{n}$ are polynomials in $R$ and can be calculated recursively by

$$
\begin{gathered}
U_{0}=0, \quad V_{0}=1, \quad a_{0}=[\sqrt{f(N)}], \\
U_{n+1}=a_{n} V_{n}-U_{n}, \quad V_{n+1}=\frac{f(N)-U_{n+1}^{2}}{V_{n}}, \\
a_{n+1}=\left[\frac{U_{n+1}+\sqrt{f(N)}}{V_{n+1}}\right] \quad(n \geq 0) .
\end{gathered}
$$

Moreover, $p(\sqrt{f(N)})=2 k$ if and only if $k$ is the smallest integer such that $U_{k} / U_{k+1} \in \mathbb{F}_{q}^{*}$, while $p(\sqrt{f(N)})=2 k+1$ if and only if $k$ is the smallest integer such that $V_{k} / V_{k+1} \in \mathbb{F}_{q}^{*}$.

The next result means that the convergents are the best approximations of $\alpha \in k_{\infty}$.

Lemma 2.1. For $\alpha \in k_{\infty}-k, P, Q \in R, Q \neq 0$ and $(P, Q)=1$, the following statements are equivalent to each other:

(1) $P / Q=P_{n} / Q_{n}$ or $P / Q=-P_{n} / Q_{n}$ for some $n \geq 0$;

(2) $\operatorname{deg}\left(P^{2}-\alpha^{2} Q^{2}\right)<\operatorname{deg} \alpha$;

(3) $\operatorname{deg}(P-\alpha Q)<-\operatorname{deg} Q$ or $\operatorname{deg}(P+\alpha Q)<-\operatorname{deg} Q$.

Proof. Let $P / Q=\left[c_{0}, c_{1}, \ldots, c_{n}\right]$ be the finite continued fraction of $P / Q$, and $P^{\prime} / Q^{\prime}=\left[c_{0}, c_{1}, \ldots, c_{n-1}\right]$. There is $\beta \in k_{\infty}$ such that

$$
\alpha=\left[c_{0}, \ldots, c_{n}, \beta\right]=\frac{\beta P+P^{\prime}}{\beta Q+Q^{\prime}} .
$$

Namely,

$$
\beta=\frac{-\alpha Q^{\prime}+P^{\prime}}{\alpha Q-P}
$$

From (2.7) and (2.3) we have

$$
\alpha-\frac{P}{Q}=\frac{(-1)^{n}}{Q\left(\beta Q+Q^{\prime}\right)}, \quad \operatorname{deg}(P-\alpha Q)=-\operatorname{deg}\left(\beta Q+Q^{\prime}\right) .
$$

Therefore

$$
\begin{gathered}
P / Q=P_{n} / Q_{n} \Leftrightarrow \operatorname{deg} \beta \geq 1 \Leftrightarrow \operatorname{deg}(P-\alpha Q)<-\operatorname{deg} Q, \\
P / Q=-P_{n} / Q_{n} \Leftrightarrow \operatorname{deg}(P+\alpha Q)<-\operatorname{deg} Q .
\end{gathered}
$$

So we proved the equivalence $(1) \Leftrightarrow(3)$. The equivalence $(2) \Leftrightarrow(3)$ is easy to prove.

The following result is analogous to Theorem $\mathrm{H}$ in [8].

Lemma 2.2. Suppose that $\xi=\left[b_{0}, b_{1}, \ldots\right] \in k_{\infty}-k$ and $\xi_{\nu}=\left[b_{\nu}, b_{\nu+1}, \ldots\right]$. Let $p, r, s \in R, \operatorname{deg} r<\operatorname{deg} s$ and $r s=d \neq 0$. If

$$
\xi^{\prime}=\frac{p \xi+r}{s}, \quad \frac{p\left[b_{0}, b_{1}, \ldots, b_{\nu-1}\right]+r}{s}=\left[d_{0}, d_{1}, \ldots, d_{\mu-1}\right],
$$


then $\xi^{\prime}=\left[d_{0}, d_{1}, \ldots, d_{\mu-1}, \xi_{\mu}^{\prime}\right]$ where $\xi_{\mu}^{\prime}=\left(p^{\prime} \xi_{\nu}+r^{\prime}\right) / s^{\prime}, p^{\prime}, r^{\prime}, s^{\prime} \in R$, $p^{\prime} s^{\prime}= \pm d, \operatorname{deg} r^{\prime}<\operatorname{deg} s^{\prime}$.

Proof. For a non-singular matrix $A=\left(\begin{array}{ll}a & b \\ c & d\end{array}\right)$ over $R$ and $\alpha \in k_{\infty}$ we define the action of $A$ on $\alpha$ by

$$
A(\alpha)=\frac{a \alpha+b}{c \alpha+d}
$$

Let $\left[b_{0}, \ldots, b_{m}\right]=A_{m} / B_{m}$ and $\left[d_{0}, \ldots, d_{l}\right]=C_{l} / D_{l}$. From (2.4) we have

$$
\begin{aligned}
\xi^{\prime} & =\frac{C_{\mu-1} \xi_{\mu}^{\prime}+C_{\mu-2}}{D_{\mu-1} \xi_{\mu}^{\prime}+D_{\mu-2}}=\left(\begin{array}{ll}
C_{\mu-1} & C_{\mu-2} \\
D_{\mu-1} & D_{\mu-2}
\end{array}\right)\left(\xi_{\mu}^{\prime}\right), \\
\xi^{\prime} & =\left(\begin{array}{ll}
p & r \\
o & s
\end{array}\right)(\xi)=\left(\begin{array}{ll}
p & r \\
o & s
\end{array}\right)\left(\begin{array}{ll}
A_{\nu-1} & A_{\nu-2} \\
B_{\nu-1} & B_{\nu-2}
\end{array}\right)\left(\xi_{\nu}\right) .
\end{aligned}
$$

Therefore $\xi_{\mu}^{\prime}=\left(\begin{array}{cc}p^{\prime} & r^{\prime} \\ t^{\prime} & s^{\prime}\end{array}\right)\left(\xi_{\nu}\right)$ where

$$
\begin{aligned}
\left(\begin{array}{ll}
p^{\prime} & r^{\prime} \\
t^{\prime} & s^{\prime}
\end{array}\right) & =\left(\begin{array}{ll}
C_{\mu-1} & C_{\mu-2} \\
D_{\mu-1} & D_{\mu-2}
\end{array}\right)^{-1}\left(\begin{array}{cc}
p & r \\
o & s
\end{array}\right)\left(\begin{array}{ll}
A_{\nu-1} & A_{\nu-2} \\
B_{\nu-1} & B_{\nu-2}
\end{array}\right) \\
& = \pm\left(\begin{array}{cc}
-D_{\mu-2} & C_{\mu-2} \\
D_{\mu-1} & -C_{\mu-1}
\end{array}\right)\left(\begin{array}{ll}
p & r \\
o & s
\end{array}\right)\left(\begin{array}{ll}
A_{\nu-1} & A_{\nu-2} \\
B_{\nu-1} & B_{\nu-2}
\end{array}\right) \\
& = \pm\left(\begin{array}{cc}
-p D_{\mu-2} & -r D_{\mu-2}+s C_{\mu-2} \\
p D_{\mu-1} & r D_{\mu-1}-s C_{\mu-1}
\end{array}\right)\left(\begin{array}{ll}
A_{\nu-1} & A_{\nu-2} \\
B_{\nu-1} & B_{\nu-2}
\end{array}\right)
\end{aligned}
$$

and

$$
\begin{aligned}
t^{\prime} & = \pm\left(p D_{\mu-1} A_{\nu-1}+r D_{\mu-1} B_{\nu-1}-s C_{\mu-1} B_{\nu-1}\right) \\
& = \pm s D_{\mu-1} B_{\nu-1}\left(\frac{p A_{\nu-1} / B_{\nu-1}+r}{s}-\frac{C_{\mu-1}}{D_{\mu-1}}\right)=0, \\
s^{\prime} & = \pm\left(p D_{\mu-1} A_{\nu-2}+r D_{\mu-1} B_{\nu-2}-s C_{\mu-1} B_{\nu-2}\right), \\
r^{\prime} & =\mp\left(p D_{\mu-2} A_{\nu-2}+r D_{\mu-2} B_{\nu-2}-s C_{\mu-2} B_{\nu-2}\right),
\end{aligned}
$$

so that $\operatorname{deg} r^{\prime}<\operatorname{deg} s^{\prime}$. From (2.8) we have

$$
p^{\prime} s^{\prime}=\left|\begin{array}{cc}
p^{\prime} & r^{\prime} \\
o & s^{\prime}
\end{array}\right|= \pm\left|\begin{array}{cc}
p & r \\
o & s
\end{array}\right|= \pm d .
$$

This completes the proof of Lemma 2.2 (cf. Theorem 3 in Chapter IV of [7]).

3. Proof of Theorem 1.1. Suppose that $N \in \widetilde{R}-E$, which means that $f(N)=A^{2} N^{2}+B N+C$ is square-free, $\sqrt{f(N)} \in k_{\infty}$ and

$$
D=B^{2}-4 A^{2} C \mid\left(2 A^{2} N+B\right)^{2} .
$$

Let

$$
\xi^{\prime}=\sqrt{f(N)}=\frac{1}{2 A} \sqrt{\left(2 A^{2} N+B\right)^{2}-D} .
$$


From (3.1) we know that there exist $U, V, W \in R$ such that

$$
2 A^{2} N+B=U V W, \quad D=U V^{2} .
$$

The formula (3.2) becomes

$$
\xi^{\prime}=\frac{V}{2 A} \xi
$$

where

$$
\begin{gathered}
\xi=\sqrt{U^{2} W^{2}-U}=[U W, \overline{2 W, 2 U W}]=\left[U W, \xi^{\prime \prime}\right], \\
\xi^{\prime \prime}=[\overline{2 W, 2 U W}]=\left[2 W, 2 U W, \xi^{\prime \prime}\right] .
\end{gathered}
$$

Now we use Lemma 2.2 repeatedly to get the continued fraction expansion of $\xi^{\prime}=\sqrt{f(N)}$. Let

$$
\eta_{1}=\frac{V U W}{2 A}=\frac{2 A^{2} N+B}{2 A}=\left[a_{0}, a_{1}, \ldots, a_{\mu_{1}-1}\right] .
$$

From (3.5) and Lemma 2.2 we have

$$
\xi^{\prime}=\left[a_{0}, a_{1}, \ldots, a_{\mu_{1}-1}, \xi_{1}^{\prime}\right]
$$

where

(3.9) $\xi_{1}^{\prime}=\frac{p_{1} \xi^{\prime \prime}+r_{1}}{s_{1}}, \quad p_{1}, r_{1}, s_{1} \in R, p_{1} s_{1}= \pm 2 A V, \operatorname{deg} r_{1}<\operatorname{deg} s_{1}$.

In general, for each $i \geq 1$ we have

$$
\xi_{i}^{\prime}=\frac{p_{i} \xi^{\prime \prime}+r_{i}}{s_{i}}, \quad p_{i}, r_{i}, s_{i} \in R, p_{i} s_{i}= \pm 2 A V, \operatorname{deg} r_{i}<\operatorname{deg} s_{i} .
$$

Let

$$
\begin{aligned}
\eta_{i+1} & =\frac{p_{i}[2 W, 2 U W]+r_{i}}{s_{i}}=\frac{p_{i}(2 W+1 /(2 U W))+r_{i}}{s_{i}} \\
& =\left[a_{\mu_{i}}, a_{\mu_{i}+1}, \ldots, a_{\mu_{i+1}-1}\right] .
\end{aligned}
$$

From (3.6) and Lemma 2.2 we have

$$
\xi_{i}^{\prime}=\left[a_{\mu_{i}}, a_{\mu_{i}+1}, \ldots, a_{\mu_{i+1}-1}, \xi_{i+1}^{\prime}\right]
$$

where

$$
\begin{array}{r}
\xi_{i+1}^{\prime}=\frac{p_{i+1} \xi^{\prime \prime}+r_{i+1}}{s_{i+1}}, \quad p_{i+1}, r_{i+1}, s_{i+1} \in R, p_{i+1} s_{i+1}= \pm 2 A V, \\
\operatorname{deg} r_{i+1}<\operatorname{deg} s_{i+1} .
\end{array}
$$

Since $A, B, C$ and $D=B^{2}-4 A^{2} C$ are fixed polynomials, we choose $N$ such that

$$
\operatorname{deg} N>\max \left\{\operatorname{deg}\left(A^{2} / B\right), \operatorname{deg}(D / A)\right\} .
$$

From (3.3) we know that

$$
\operatorname{deg}(U V W)=\operatorname{deg}\left(A^{2} N\right)>\operatorname{deg}(A D)=\operatorname{deg}\left(A U V^{2}\right)
$$


so that $\operatorname{deg} W>\operatorname{deg}(A V)=\operatorname{deg}\left(p_{i} s_{i}\right) \geq \operatorname{deg} s_{i}(i \geq 1)$. Therefore by (3.11),

$$
\operatorname{deg} a_{\mu_{i}}=\operatorname{deg}\left[\eta_{i+1}\right]=\operatorname{deg} \frac{p_{i} W}{s_{i}} \geq 1 \quad(i \geq 1)
$$

and by (3.8) and (3.11) we have the continued fraction expansion of $\xi^{\prime}=$ $\sqrt{f(N)}$ :

$$
\begin{aligned}
& \sqrt{f(N)} \\
& =\left[a_{0}, \ldots, a_{\mu_{1}-1}, a_{\mu_{1}}, \ldots, a_{\mu_{2}-1}, \ldots, a_{\mu_{i}}, a_{\mu_{i}+1}, \ldots, a_{\mu_{i+1}}, \ldots\right] .
\end{aligned}
$$

The total number of tuples $(p, r, s)$ satisfying $p s= \pm 2 A N$ and $\operatorname{deg} r<$ $\operatorname{deg} s$ is at most $M=q^{2 \operatorname{deg} A N+1}$, thus from (3.10) we know that there exist $l$ and $j, 1 \leq l<j \leq M$, such that $\xi_{l}^{\prime}=\xi_{j}^{\prime}$. From the expansion (3.15) we know that

$$
p(\sqrt{f(N)}) \leq \sum_{i=l}^{j-1}\left(\mu_{i+1}-\mu_{i}\right) .
$$

We use Lemma 2.2 again to estimate $\mu_{l}$. Let

$$
\frac{2 W p_{i}+r_{i}}{s_{i}}=\left[c_{0}, c_{1}, \ldots, c_{t-1}\right] .
$$

By Lemma 2.2 and (3.11) we have

$$
\eta_{i+1}=\left[c_{0}, c_{1}, \ldots, c_{t-1}, \frac{p^{\prime}(2 U W)+r^{\prime}}{s^{\prime}}\right]
$$

where $p^{\prime} s^{\prime}= \pm 2 A V$ and $\operatorname{deg} r^{\prime}<\operatorname{deg} s^{\prime}$, so that $\operatorname{deg}\left(\sqrt{p^{\prime}(2 U W)+r^{\prime}} / s^{\prime}\right) \geq$ 1. Let

$$
\frac{p^{\prime}(2 U W)+r^{\prime}}{s^{\prime}}=\left[c_{t}, c_{t+1}, \ldots, c_{t+\lambda-1}\right] .
$$

Then $\eta_{i+1}=\left[c_{0}, c_{1}, \ldots, c_{t-1}, c_{t}, \ldots, c_{t+\lambda-1}\right]$ and $\mu_{i+1}-\mu_{i}=t+\lambda$ by (3.11). From the recursive formula for $Q_{i}$ in $(2.2)$ and $\operatorname{deg} c_{i} \geq 1(0 \leq i \leq t+\lambda-1)$ we know that

$$
t \leq \operatorname{deg} s_{i} \leq \operatorname{deg}(A V), \quad \lambda \leq \operatorname{deg} s^{\prime} \leq \operatorname{deg}(A V) .
$$

Therefore $\mu_{i+1}-\mu_{i}=t+\lambda \leq 2 \operatorname{deg}(A V)$ and by (3.16),

$$
p(\sqrt{f(N)}) \leq(j-l) 2 \operatorname{deg}(A V) \leq 2 M \operatorname{deg}(A V) \leq 2 \operatorname{deg} \sqrt{(A V) q^{2 \operatorname{deg}(A V+1)}}
$$

provided the formula (3.14) is satisfied. Since there are only finitely many $N$ such that $\operatorname{deg} N \leq \max \left\{\operatorname{deg}\left(A^{2} / B\right), \operatorname{deg}(D / A)\right\}$, this completes the proof of Theorem 1.1.

4. Proof of Theorem 1.2. Now we assume that $f(N)=A^{2} N^{2}+B N+C$ and $D=B^{2}-4 A^{2} C \nmid\left(2 A^{2} N+B\right)^{2}$. Let $l=p(\sqrt{f(N)})$ and

$$
\sqrt{f(N)}=\left[a_{0}, a_{1}, \ldots, a_{n}, \ldots\right]
$$




$$
\begin{aligned}
\alpha_{i} & =\left[a_{i}, a_{i+1}, \ldots\right], \quad P_{i} / Q_{i}=\left[a_{0}, a_{1}, \ldots, a_{i}\right], \\
\varphi_{i} & =P_{i}+Q_{i} \sqrt{f(N)}, \quad \bar{\varphi}_{i}=P_{i}-Q_{i} \sqrt{f(N)} .
\end{aligned}
$$

Then $\varepsilon=\varphi_{l-1}$ is the fundamental unit of the quadratic function field $K=$ $k(\sqrt{f(N)})$. Let

$$
G=(A, B), \quad \beta=D G^{-2}, \quad U=2 A G^{-1}, \quad V=B G^{-1} .
$$

We choose

$$
A_{1}=A U N+V=\left(2 A^{2} N+B\right) G^{-1}, \quad B_{1}=U=2 A G^{-1}
$$

and

$$
X=A_{1}+B_{1} \sqrt{f(N)} .
$$

It is easy to see that

$$
\operatorname{Norm}(X)=X \bar{X}=A_{1}^{2}-B_{1}^{2} f(N)=\beta
$$

where Norm denotes the norm for $\mathbb{F}_{q}(t, \sqrt{f(N)}) / \mathbb{F}_{q}(t)$. For each $k \geq 1$, let

$$
X^{k}=A_{k}+B_{k} \sqrt{f(N)}, \quad A_{k}, B_{k} \in R .
$$

The polynomials $A_{k}$ and $B_{k}$ can be calculated recursively by

$$
A_{k+1}=A_{1} A_{k}+B_{1} B_{k} f(N), \quad B_{k+1}=A_{1} B_{k}+B_{1} A_{k} .
$$

Let

$$
D_{k}=\left(A_{k}, B_{k}\right), \quad A_{k}^{\prime}=A_{k} / D_{k}, \quad B_{k}^{\prime}=B_{k} / D_{k}, \quad N_{k}=A_{k}^{\prime 2}-B_{k}^{\prime} f(N) .
$$

Then $\left(A_{k}^{\prime}, B_{k}^{\prime}\right)=1$. Finally we choose

$$
M=\left[\frac{\operatorname{deg} f(N)}{2 \operatorname{deg} \beta}\right]-1 .
$$

Lemma 4.1. For $1 \leq k \leq M$, there exists $i_{k}$ such that $A_{k}^{\prime} / B_{k}^{\prime}=P_{i_{k}} / Q_{i_{k}}$.

Proof. We have

$$
\begin{aligned}
\operatorname{deg}\left(A_{k}^{\prime 2}-B_{k}^{\prime 2} f(N)\right) & =\operatorname{deg}\left(A_{k}^{2}-B_{k}^{2} f(N)\right)-2 \operatorname{deg} D_{k} \\
& \leq \operatorname{deg}\left(A_{k}^{2}-B_{k}^{2} f(N)\right)=\operatorname{deg}\left(\operatorname{Norm}\left(X^{k}\right)\right)=k \operatorname{deg} \beta \\
& \leq M \operatorname{deg} \beta<\frac{1}{2} \operatorname{deg} f(N) .
\end{aligned}
$$

Then the conclusion follows from Lemma 2.1 and $\operatorname{deg}\left(A_{k}^{\prime}+B_{k}^{\prime} \sqrt{f(N)}\right) \geq 0$.

LEMMA 4.2. $i_{k+1}-i_{k} \geq 2$ for $1 \leq k \leq M-1$.

Proof. From the condition $D \nmid\left(2 A^{2} N+B\right)^{2}$ we know that there is an irreducible polynomial $P=P(t)$ in $R$ such that $v_{P}(D)>2 v_{P}\left(2 A^{2} N+B\right)$, which means that $v_{P}(\beta)>2 v_{P}\left(A_{1}\right)$ where $v_{P}$ is the normal $P$-adic exponential valuation. From $\beta=\operatorname{Norm}(X)=A_{1}^{2}-B_{1}^{2} f(N)$ we have $v_{P}\left(A_{1}^{2}\right)=$ $v_{P}\left(B_{1}^{2} f(N)\right)$. Then $\left(A_{1}, B_{1}\right)=1$ and $f(N) \in \widetilde{R}$ imply that

$$
v_{P}\left(B_{1}\right)=v_{P}(f(N))=v_{P}\left(A_{1}\right)=0 .
$$


Now we prove that $v_{P}\left(A_{k}\right)=v_{P}\left(B_{k}\right)=0$ for all $k \geq 1$ by induction. This is true for $k=1$ from (4.4). Assume that $v_{P}\left(B_{i}\right)=v_{P}\left(A_{i}\right)=0$ for some $i \geq 1$. Since $v_{P}(f(N))=0, v_{P}(\beta)>2 v_{P}\left(A_{1}\right)=0$ and $\beta^{i}=$ $A_{i}^{2}-B_{i}^{2} f(N)$, we know that $A_{i}^{2} \equiv B_{i}^{2} f(N)(\bmod P)$, which means that $\left(\frac{f(N)}{P}\right)=1$ and $P$ is split in the quadratic field $K=\mathbb{F}_{q}(t)(\sqrt{f(N)})$ where $(\bar{P})$ means the Legendre symbol. Let $P=p \widetilde{p}$ be the decomposition in $K$. Then $X=A_{1}+B_{1} \sqrt{f(N)}$ has to be divisible by $p$ or $\widetilde{p}$. We can assume that

$$
A_{1}+B_{1} \sqrt{f(N)} \equiv 0(\bmod p) .
$$

From $v_{P}\left(B_{1}\right)=0$ we know that $v_{p}\left(B_{1}\right)=0$ so that

$$
\frac{A_{1}}{B_{1}} \equiv-\sqrt{f(N)}(\bmod p)
$$

Moreover, $A_{i}+B_{i} \sqrt{f(N)}=\left(A_{1}+B_{1} \sqrt{f(N)}\right)^{i} \equiv 0(\bmod p)$. From the assumption $v_{P}\left(B_{i}\right)=0$ we have

$$
A_{i} / B_{i} \equiv-\sqrt{f(N)}(\bmod p) .
$$

Then from (4.1) we see that

$$
\frac{B_{i+1}}{B_{1} B_{i}}=\frac{A_{1}}{B_{1}}+\frac{A_{i}}{B_{i}} \equiv-2 \sqrt{f(N)} \not \equiv 0(\bmod p),
$$

which means that $v_{p}\left(B_{i+1}\right)=0$ so that $v_{P}\left(B_{i+1}\right)=0$. Finally we deduce that $v_{P}\left(A_{i+1}\right)=0$ from $\beta^{i+1}=A_{i+1}^{2}-B_{i+1}^{2} f(N)$. Thus we have proved that $v_{P}\left(A_{k}\right)=v_{P}\left(B_{k}\right)=0$ for all $k \geq 1$. Then $v_{P}\left(D_{k}\right)=0$ since $D_{k}=\left(A_{k}, B_{k}\right)$. From (2.3) and (2.4) we have

$$
\sqrt{f(N)}-\frac{P_{n}}{Q_{n}}=\frac{(-1)^{n+1}}{Q_{n}\left(\alpha_{n+1} Q_{n}+Q_{n-1}\right)} .
$$

Therefore

$$
\begin{aligned}
\operatorname{deg} \bar{\varphi}_{n} & =\operatorname{deg}\left(P_{n}-Q_{n} \sqrt{f(N)}\right)=-\operatorname{deg}\left(\alpha_{n+1} Q_{n}+Q_{n-1}\right) \\
& =-\operatorname{deg}\left(a_{n+1} Q_{n}+Q_{n-1}\right)=-\operatorname{deg} Q_{n+1} .
\end{aligned}
$$

From $\operatorname{deg}\left(A_{1}-B_{1} \sqrt{f(N)}\right)<0$ and $D_{k} \mid D_{k+1}$ (by definition) we know that

$$
\begin{aligned}
-\operatorname{deg} Q_{i_{k}+1} & =\operatorname{deg} \bar{\varphi}_{i_{k}}=\operatorname{deg}\left(P_{i_{k}}-Q_{i_{k}} \sqrt{f(N)}\right) \\
& =\operatorname{deg}\left(A_{k}^{\prime}-B_{k}^{\prime} \sqrt{f(N)}\right) \\
& =-\operatorname{deg} D_{k}+\operatorname{deg}\left(A_{k}-B_{k} \sqrt{f(N)}\right) \\
& =-\operatorname{deg} D_{k}+k \operatorname{deg}\left(A_{1}-B_{1} \sqrt{f(N)}\right) \\
& >-\operatorname{deg} D_{k+1}+(k+1) \operatorname{deg}\left(A_{1}-B_{1} \sqrt{f(N)}\right) \\
& =\operatorname{deg} \bar{\varphi}_{i_{k+1}}=-\operatorname{deg} Q_{i_{k+1}+1}
\end{aligned}
$$


Therefore $\operatorname{deg} Q_{i_{k+1}+1}>\operatorname{deg} Q_{i_{k}+1}$ which means that $i_{k+1}>i_{k}$. If $i_{k+1}=$ $i_{k}+1$, then

$$
P_{i_{k+1}} Q_{i_{k}}-P_{i_{k}} Q_{i_{k+1}}=P_{i_{k}+1} Q_{i_{k}}-P_{i_{k}} Q_{i_{k}+1}=(-1)^{i_{k}+1},
$$

so that $v_{P}\left(P_{i_{k+1}} Q_{i_{k}}-P_{i_{k}} Q_{i_{k+1}}\right)=0$. On the other hand,

$$
\begin{aligned}
& v_{P}\left(P_{i_{k+1}} Q_{i_{k}}-P_{i_{k}} Q_{i_{k+1}}\right)= v_{P}\left(A_{k+1}^{\prime} B_{k}^{\prime}-A_{k}^{\prime} B_{k+1}^{\prime}\right) \\
&= v_{P}\left(A_{k+1} B_{k}-A_{k} B_{k+1}\right) \\
& \quad\left(\text { since } v_{P}\left(D_{k}\right)=v_{P}\left(D_{k+1}\right)=0\right) \\
&=v_{P}\left(\left(A_{k} A_{1}+B_{k} B_{1} f(N)\right) B_{k}\right. \\
&\left.-A_{k}\left(A_{k} B_{1}+A_{1} B_{k}\right)\right) \\
&= v_{P}\left(B_{1}\left(A_{k}^{2}-B_{k}^{2} f(N)\right)\right)=v_{P}\left(\beta^{k}\right) \\
& \quad \quad\left(\text { since } v_{P}\left(B_{1}\right)=0\right) \\
&= k v_{P}(\beta)>0 \quad(\text { for } k \geq 1) .
\end{aligned}
$$

This contradiction shows that $i_{k+1}-i_{k} \geq 2$, which completes the proof of Lemma 4.2 .

LEMma 4.3. Let

$$
M_{1}=\left[\frac{\operatorname{deg} f(N)-2 \operatorname{deg} U}{2 \operatorname{deg} \beta}\right]-1(\leq M) .
$$

Then $\operatorname{deg} \varphi_{i_{k}}<\operatorname{deg} \varepsilon$ for all $1 \leq k \leq M_{1}$ where $\varepsilon=\varphi_{l-1}$ is the fundamental unit in $K=\mathbb{F}_{q}(t, \sqrt{f(N)})$.

Proof. If $\operatorname{deg} \varphi_{i_{s}} \geq \operatorname{deg} \varepsilon$ for some $s\left(1 \leq s \leq M_{1}\right)$, let $s$ be the smallest one satisfying this condition. Then

$$
\varphi_{i_{s}} \bar{\varepsilon}=\left(P_{i_{s}}+Q_{i_{s}} \sqrt{f(N)}\right)\left(P_{l-1}-Q_{l-1} \sqrt{f(N)}\right)=A^{\prime}+B^{\prime} \sqrt{f(N)}
$$

where $A^{\prime}, B^{\prime} \in R$, and

$$
\operatorname{deg}\left(A^{\prime 2}-B^{\prime 2} f(N)\right)=\operatorname{deg}\left(\operatorname{Norm}\left(\varphi_{i_{s}}\right)\right)=\operatorname{deg}\left(\operatorname{Norm}\left(\varphi_{i_{s}} \bar{\varepsilon}\right)\right)<\frac{1}{2} \operatorname{deg} f(N) .
$$

From Lemma 2.1 and $\operatorname{deg}\left(\varphi_{i_{s}} \bar{\varepsilon}\right)=\operatorname{deg} \varphi_{i_{s}}-\operatorname{deg} \varepsilon \geq 0$ we know that there exists $j$ such that $\varphi_{i_{s}} \bar{\varepsilon}=\alpha \varphi_{j}$ for some $\alpha \in \mathbb{F}_{q}^{*}$. Therefore $D_{s}^{-1} X^{s}=\varphi_{i_{s}}=$ $\alpha^{\prime} \varepsilon \varphi_{j}\left(\alpha^{\prime} \in \mathbb{F}_{q}^{*}\right)$. If $j \geq i_{1}$, then

$$
s \operatorname{deg} X \geq \operatorname{deg}\left(D_{s}^{-1} X^{s}\right)=\operatorname{deg}\left(\varepsilon \varphi_{j}\right) \geq \operatorname{deg}\left(\varepsilon \varphi_{i_{1}}\right)=\operatorname{deg}(\varepsilon X)>\operatorname{deg} X>0 .
$$

Thus $s \geq 2$ and

$$
\operatorname{deg} \varphi_{i_{s-1}} \geq \operatorname{deg}\left(D_{s-1} D_{s}^{-1} \varphi_{i_{s-1}}\right)=\operatorname{deg}\left(D_{s}^{-1} X^{s-1}\right) \geq \operatorname{deg} \varepsilon
$$

which contradicts the definition of $s$. Therefore $j<i_{1}$. Since $\operatorname{deg}\left(\varphi_{j} \bar{\varphi}_{j}\right)=\operatorname{deg}\left(\varphi_{i_{s}} \bar{\varphi}_{i_{s}}\right)=\operatorname{deg}\left(\beta^{s} D_{s}^{-2}\right), \quad \bar{\varphi}_{j}=(-1)^{j+1}\left(\alpha_{j+1} Q_{j}+Q_{j-1}\right)^{-1}$ 
and $\operatorname{deg} \alpha_{j+1}=\operatorname{deg} a_{j+1}$, we know that $\operatorname{deg} \bar{\varphi}_{j}=-\operatorname{deg} Q_{j+1}=-\operatorname{deg} Q_{i_{1}}=$ $-\operatorname{deg} U$ and

$$
\operatorname{deg}\left(\beta^{s} U\right) \geq \operatorname{deg}\left(\beta^{s} D_{s}^{-2} U\right) \geq \operatorname{deg}\left(\beta^{s} D_{s}^{-2} \bar{\varphi}_{j}^{-1}\right)=\operatorname{deg} \varphi_{j} \geq \frac{1}{2} \operatorname{deg} f(N) .
$$

Therefore $s \geq(\operatorname{deg} f(N)-2 \operatorname{deg} U) /(2 \operatorname{deg} \beta)$, which contradicts $s \leq M_{1}$. This completes the proof of Lemma 4.3.

Now Theorem 1.2 is a direct consequence since Lemma 4.2 says that $i_{k+1}-i_{k} \geq 2$ for $1 \leq k \leq M_{1}-1$ and Lemma 4.3 says that $1 \leq i_{k} \leq$ $p(\sqrt{f(N)})$ for all $1 \leq k \leq M_{1}$, so that

$p(\sqrt{f(N)}) \geq 2 M_{1}-1=2\left[\frac{\operatorname{deg} f(N)-2 \operatorname{deg} U}{2 \operatorname{deg} \beta}\right]-1 \rightarrow \infty \quad$ as $\operatorname{deg} N \rightarrow \infty$.

This completes the proof of Theorem 1.2.

Example. Let $f(x)=x^{2}+C$ where $C=2 a+1, a \in R, \operatorname{deg} a \geq 1$. We have $A=1, B=0, \beta=-4 C=D$ and $U=1$. For each $k \geq 2$, we choose $N=C^{k}+a$ so that $f(N)=\left(C^{k}+a\right)^{2}+C$. Since $D=-4 C \nmid\left(2 A^{2} N+B\right)^{2}=$ $4\left(C^{k}+a\right)^{2}, N$ belongs to the set $E$ for all $k \geq 2$. We use Fact 2 to determine the quasi-period $p(\sqrt{f(N)})$ as follows:

$$
\begin{aligned}
& U_{0}=0, \quad V_{0}=1, \quad a_{0}=[\sqrt{f(N)}]=C^{k}+a, \\
& U_{1}=C^{k}+a, \quad V_{1}=C, \quad a_{1}=\left[\frac{2 C^{k}+2 a}{C}\right]=\left[\frac{2 C^{k}+C-1}{C}\right]=2 C^{k-1}+1, \\
& U_{2}=C^{k}+a+1, \quad V_{2}=-2 C^{k-1}, \quad a_{2}=\left[\frac{2 C^{k}+C}{-2 C^{k-1}}\right]=-C, \\
& U_{3}=C^{k}-a-1, \quad V_{3}=\frac{C+\left(2 C^{k}-1\right) C}{-2 C^{k-1}}=-C^{2}, \\
& a_{3}=\left[\frac{2 C^{k}-1}{-C^{2}}\right]=-2 C^{k-2}, \\
& U_{4}=C^{k}+a+1, \quad V_{4}=2 C^{k-2}, \quad a_{4}=C^{2}, \\
& U_{5}=C^{k}-a-1, \quad V_{5}=C^{3}, \quad a_{5}=2 C^{k-3},
\end{aligned}
$$

In general, $V_{2 i+1}=\alpha C^{i+1}, V_{2 i}=\alpha^{\prime} C^{k-i}$, where $\alpha, \alpha^{\prime} \in \mathbb{F}_{q}^{*}$. If $k=2 n$, then $V_{2 n} / V_{2 n+1} \in \mathbb{F}_{q}^{*}$ and $p(\sqrt{f(N)})=4 n+1=2 k-1$. If $k=2 n$, then $V_{2 n-1} / V_{2 n} \in \mathbb{F}_{q}^{*}$ and $p(\sqrt{f(N)})=4 n-1=2 k-1$. Therefore $p(\sqrt{f(N)})=$ $2 k-1$ for all $k \geq 2$. On the other hand,

$$
2 M_{1}-1=2\left[\frac{\operatorname{deg} f(N)-2 \operatorname{deg} U}{2 \operatorname{deg} \beta}\right]-1=\frac{2 \operatorname{deg}\left(C^{k}+a\right)}{\operatorname{deg} C}-1=2 k-1 .
$$


Therefore $p(\sqrt{f(N)})=2 M_{1}-1$, which shows that our general estimate $p(\sqrt{f(N)}) \geq 2 M_{1}-1$ is the best one.

Acknowledgments. I would like to thank an anonymous referee for several suggestions that improved the exposition of this paper.

\section{References}

[1] E. Artin, Quadratische Körper im Gebiet der höheren Kongruenzen I, II, Math. Z. 19 (1924), 154-246.

[2] A. F arhane, Minoration de la période du développement de $\sqrt{a^{2} n^{2}+b n+c}$ en fraction continue, Acta Arith. 67 (1994), 63-67.

[3] C. D. González, Class number of quadratic function fields and continued fractions, J. Number Theory 40 (1992), 38-59.

[4] D. Hayes, Real quadratic function fields, in: CMS Conf. Proc. 7, Amer. Math. Soc., 1987, 203-236.

[5] L. K. Hua, Introduction to Number Theory, Springer, 1982.

[6] S. Louboutin, Une version effective d'un théorème de A. Schinzel sur longueurs des périodes de certains développements en fractions continues, C. R. Acad. Sci. Paris Sér. I 308 (1989), 511-513.

[7] B. de Mathan, Approximations diophantiennes dans un corps local, Bull. Soc. Math. France Mém. 21 (1970).

[8] A. Schinzel, On some problems of the arithmetical theory of continued fractions, Acta Arith. 6 (1961), 393-413.

The Fundamental Science Department

Nanjing Agriculture College

Nanjing 210038, China

E-mail: binhuang@public1.ptt.js.cn

Received on 22.5.1997

and in revised form on 12.4.1999 